\title{
The digestion of fibre by pigs
}

\section{Effects of the amount and type of fibre on physical characteristics of segments of the gastrointestinal tract}

\author{
By GEORGE STANOGIAS* AND G. R. PEARCE† \\ School of Agriculture and Forestry, University of Melbourne, Parkville, \\ Victoria 3052, Australia
}

(Received 18 September 1984 - Accepted 20 November 1984)

\begin{abstract}
1. The aim of the study was to determine the relative effects of feeding growing pigs with graded amounts of neutral-detergent fibre (NDF) from various sources on the empty wet weight of segments of the pig gastrointestinal tract, on the weight and moisture content of their digesta, and on the pattern of digesta movement in them.

2. Increased NDF intakes were associated with significantly higher wet weights of all gastrointestinal segments and increased lengths of the caecum. The lengths of both the small and the large intestines were unaffected by the ingestion by the pigs of different amounts of NDF from various sources. However, the caecum responded to these increased intakes of NDF by significant increases in length. The source of NDF in the diet was a factor that markedly influenced both the length and the weight of the distal colon. The nature of these increases in weight and length morphologically and their biological significance have not been determined.

3. Despite some significant differences, neither the type nor the level of dietary NDF had any appreciable effect on the dry weight and on the proportion of dry matter (DM) of the contents in the stomach. As the level of NDF intake was increased, more undigested dietary material was found in all segments of the digestive tract of the pigs. The proportion of DM in the residues decreased progressively from the caecal contents to the contents of the distal colon. In most cases the degree to which the level of NDF intake affected the weight of the contents and the proportion of DM in them was highly dependent on the source of NDF in the diet.

4. The distribution of the feed consumed in the morning and in the evening, as measured with stained feed particles and polyethylene beads, was extremely variable. It is concluded that prolonged intakes by pigs of diets containing high levels of fibre may lead to a hypertrophy and hence increased weight of segments of the gastrointestinal tract.
\end{abstract}

It has been suggested that pigs adapt themselves to feeding on high-fibre, low-energy diets by increasing their food intakes in an effort to compensate for the low energy value of the diet (Cole et al. 1967; Baird et al. 1970, 1975; Kass et al. 1980). In the pig (Wussow \& Weniger, 1954; Bohman et al. 1955), as well as in the rat (Wierda, 1950; Dowling et al. 1967), such an adaptation is achieved by an enlargement of some or all organs of the gastrointestinal tract. Thus, Bohman et al. (1955) and Kass et al. (1980) reported an increase in the weight of the colon of pigs fed on diets containing from 200 to $600 \mathrm{~g}$ lucerne (Medicago sativa) $/ \mathrm{kg}$, while in rats, increased intake of bulky diets resulted in increased length or weight, or both, of either the small intestine (Friedman, 1953; Younoszai et al. 1978; Brown et al. 1979) or large intestine (Fischer, 1957; Dowling et al. 1967; Brown et al. 1979). It is a widely accepted view that these changes in the various segments of the gastrointestinal tract are related to properties of the fibrous material such as its water-holding capacity, its physical bulk and its chemical composition. In this regard, it might be reasonable to assume that different types of fibre will affect gastrointestinal responses differently.

The retention time of digesta in the caecum and the large intestine is of particular importance for the pig as these organs represent the only sites in which digestion of dietary fibre can occur. However, there is limited information on the effects of increased intakes of fibre with varying chemical composition on the weight and pattern of digesta movement along the gastrointestinal tract of the pig.

* Present address: Livestock Institute, Giannitsa, Greece.

$\dagger$ For reprints. 
The purpose of the present investigation was to assess, in pigs, the effect of feeding graded levels of neutral-detergent fibre (NDF) from a wide range of fibre sources with varying physical and chemical properties, on gross visual changes (e.g. lesions) of segments of the gastrointestinal tract, their empty wet weights and the dry weights and the pattern of distribution of digesta in them.

\section{MATERIALS AND METHODS}

\section{Animals and diets}

The results were obtained from the pigs used in a digestibility and rate of passage study which has been reported earlier (Stanogias \& Pearce, 1985). In this, groups of entire male pigs weighing 45 (SE 2) $\mathrm{kg}$ were maintained in metabolism cages and given a basal, non-fibre diet with four levels of NDF from eight different sources, i.e. soya-bean hulls, lupin (Lupinus sp.) hulls, pea (Pisum sativum) hulls, wheat bran, maize hulls, maize cobs, oat hulls and lucerne stems. The NDF content of these diets was $75,150,225$ and $300 \mathrm{~g} / \mathrm{kg}$ and the daily allocation of feed in the pigs assigned to each of the previously-mentioned diets provided them with $69,151,249$ or $367 \mathrm{~g}$ NDF respectively. At the conclusion of the digestibility and rate of passage measurements, the pigs, excluding the control (basal diet only) group, were slaughtered for the pupose of examining features of their digestive tracts and contents. Measurements on the contents of segments of the tract were made on all groups, but measurements of the weights and lengths of segments were made only on the groups that received lupin hulls, wheat bran, maize cobs and lucerne stems. Details of the experimental design have been described by Stanogias \& Pearce (1985).

\section{Measurements on the gastrointestinal tract}

Soon after slaughter, the entire gastrointestinal tract from each pig was removed from the abdominal cavity and separated immediately by ligature into five segments: stomach, small intestine, caecum, proximal colon, distal colon plus rectum which will be referred to as distal colon. Because pieces of flesh of varying weights from the area around the rectum were always associated with the end of the rectum as a result of the degutting procedure, the final $50 \mathrm{~mm}$ of the rectum was always removed before measuring the length and the weight of this part of the alimentary tract. The proximal and the distal parts of the remaining colon were obtained by dividing it into two parts equal in length. After removing the mesentery and adhering fat the length of each segment was measured. Following this, each segment was opened with the aid of a pair of scissors and the digesta were emptied into preweighed aluminium pans. The weight of each segment was then measured and after washing and drying it on a dry cloth its weight was measured again. The difference in the two weights was attributed to digesta that could not be removed by the earlier procedure, although the possibility that some gut mucoproteins were contributing to this weight cannot be excluded. The weight of the digesta from each segment was also measured and samples were taken for the determination of dry matter (DM) and for the number of stained feed particles and polyethylene beads, used to determine the proportionate distribution of digesta. Digesta DM was determined by drying samples to constant weight in a forced-draught oven at $105^{\circ}$

\section{Distribution of digesta in the alimentary tract}

To investigate the effect of the level and the type of dietary NDF intake on the distribution of digesta within the alimentary tract, stained fibre particles were included in the morning and evening feeds of the day before slaughter. The particles given in the morning were stained red and those given in the evening were stained green. In the experiments in which 
lupin hulls, maize cobs, wheat bran and lucerne stems were given, in addition to the stained particles, a known amount of polyethylene beads was also given with the evening meal. Both the fibre-staining procedure and amount of markers used were the same as described by Stanogias \& Pearce (1985). The numbers of particles and beads found in each segment were expressed as a proportion of the total from all segments.

\section{Statistical analysis}

The values were statistically analysed as either a $4 \times 8$ or a $4 \times 4$ factorial design. The two factors were the source of fibre in the diet (FS) and the level of NDF content in the diet with orthogonal (NDF), linear $\left(\mathrm{NDF}_{\mathrm{L}}\right)$ and quadratic $\left(\mathrm{NDF}_{\mathrm{Q}}\right)$ effects. The sum of squares for the interaction between fibre source and level of NDF in the diet were also split into linear $\left(\mathrm{FS} \times \mathrm{NDF}_{\mathrm{L}}\right)$ and quadratic $\left(\mathrm{FS} \times \mathrm{NDF}_{\mathrm{Q}}\right)$ components. These statistical procedures were used as outlined by Snedecor $\&$ Cochran (1973).

\section{RESULTS}

\section{Weights and lengths of gastrointestinal segments}

Measurements of the wet weights and lengths of individual or combined segments of the gastrointestinal tract are summarized in Table 1.

The wet weight of the stomach of the pigs on the different treatments increased linearly $(P<0.01)$ with the level of NDF in the diet. The average stomach weight over all levels of NDF did differ significantly $(P<0.001)$ among sources of NDF in the diet. There was no evidence of interaction between level and sources of NDF in the diet.

The dominant effect on the wet weight of the small intestine of the level of NDF intake by the pigs was the linear $(P<0.001)$ increase in the weight of the intestine with the amount of NDF ingested. However, there was some evidence of curvilinearity $(P<0 \cdot 05)$. A significant interaction was found between source of NDF and both the linear and the quadratic term of NDF level $(P<0.001)$, in intestine wet weight. The overall analysis of variance indicated that the length of the small intestine was significantly $(P<0.001)$ affected by the source of NDF in the diet but not by the level of NDF in the diet. However, when the interaction between source and level of NDF in the diet was partitioned and tested for differences in linear and quadratic regressions on levels of NDF intake among the groups of pigs fed on different types of fibres, significant differences of both the linear $(P<0.05)$ and the quadratic $(P<0.001)$ components were found.

Both wet weight and length of the caecum were increased linearly $(P<0.001)$ with the level of NDF intake by the pigs. There was no evidence of any curvilinearity. Also, the source of NDF in the diet had a significant $(P<0.001)$ effect on both measurements and this effect was independent of the level of NDF in the diet.

The wet weight of the proximal and the distal colons were increased linearly $(P<0.001)$ as a result of increased NDF intakes although, in the case of the distal colon, there was some evidence of curvilinearity $(P<0 \cdot 05)$. Also, although the analysis of variance showed that the source of NDF in the diet had no effect on the wet weight of the distal colon, partitioning of the interaction between level and source of NDF in the diet revealed significant interactions between source of NDF and the linear component of NDF level in both the proximal $(P<0.001)$ and the distal $(P<0.01)$ colon wet weights.

The length of the distal colon was unaffected by the quantity of NDF ingested but it was significantly $(P<0.001)$ influenced by the source of NDF in the diet. 


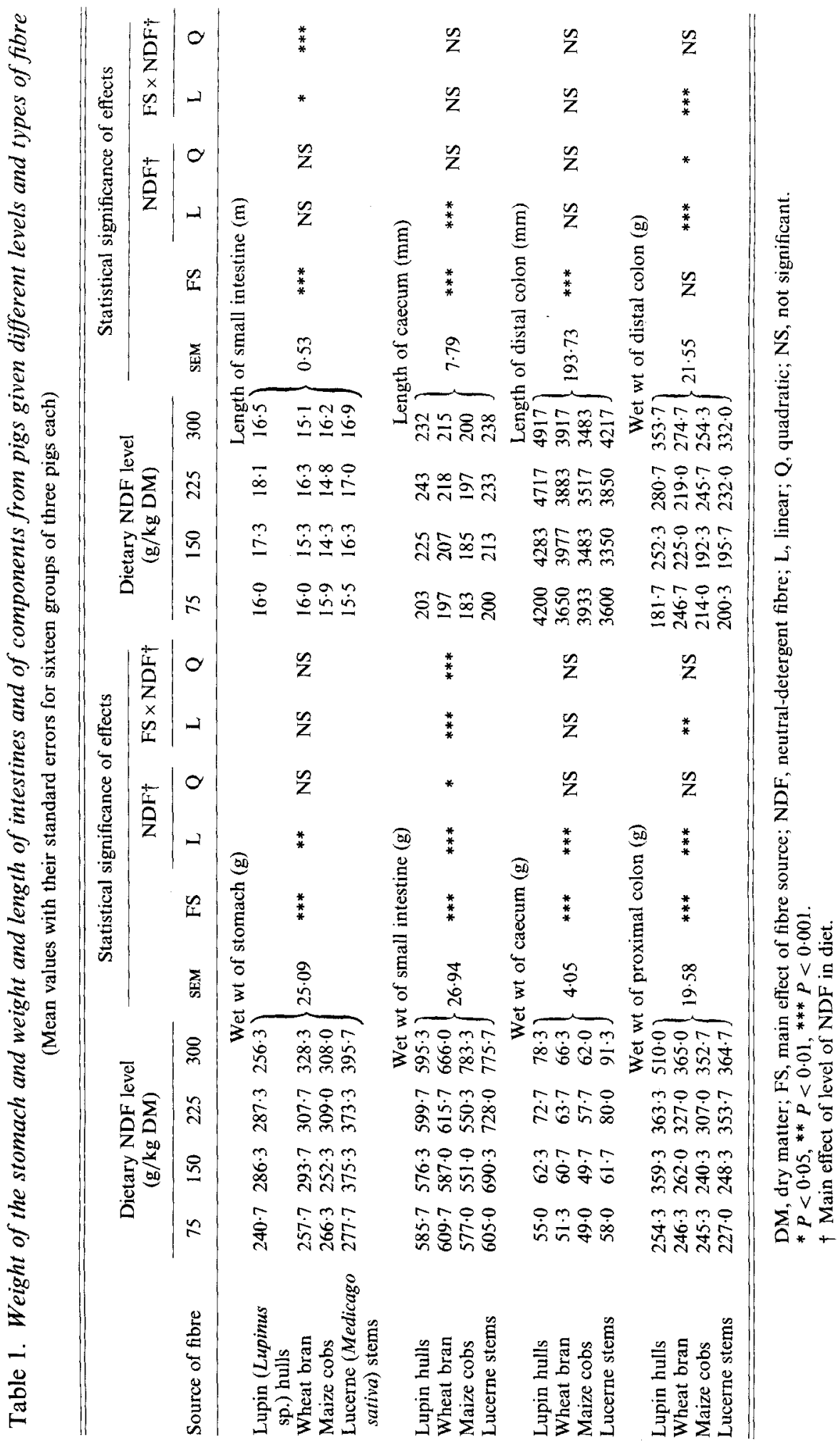




\section{Measurement of the contents of the gastrointestinal organs}

Values for the dry weight and the proportion of DM in the contents in the segments of the gastrointestinal organs are presented in Table 2.

Over all types of NDF in the diet both the dry weight and the proportion of DM of the contents in the stomach increased with the NDF intake by the pigs $(P<0.001)$. There was a large linear component to this increase $(P<0.001)$. Only the dry weight of the contents in the stomach was affected by the source of NDF in the diets and this effect was independent of the level of NDF in the diet.

The dry weight and the proportion of DM in the contents of the small intestine were increased linearly $(P<0.001$ or $P<0.05)$ with increasing levels of NDF intake although, in the case of the proportion of DM in the contents, there was some evidence of curvilinearity $(P<0.01)$. However, this increase in both the dry weight and the proportion of DM in the contents with increasing intakes of NDF were highly related to the source of NDF in the diet since a highly significant interaction between NDF source and both the linear $(P<0.001)$ and the quadratic $(P<0.001$ or $P<0.01)$ components of the NDF level occurred.

The dry weight of the contents in the caecum increased linearly $(P<0.001)$ whereas the proportion of DM in the same contents decreased linearly $(P<0.001)$ with increasing levels of NDF intake by the pigs. The source of NDF had a large influence $(P<0.001)$ on these response criteria too. However, only for the dry weight of the contents in the caecum was there evidence of an interrelation between the two main effects, as a significant interaction between NDF source and both the linear $(P<0.001)$ and the quadratic $(P<0.05)$ components of the NDF level.

Variation in the level of NDF intake by the pigs and the source of NDF in the diet had a highly significant $(P<0.001)$ effect on the dry weight of the contents in both parts of the colon. Increased NDF intakes were associated with higher dry weights of colon contents, the influence of NDF intake being linear $(P<0.001)$ although there was evidence of curvilinearity $(P<0.001$ or $P<0.05)$. A significant interaction was found between source of NDF in the diet and both the linear $(P<0.001)$ and the quadratic $(P<0.001$ or $P<0.01)$ components of the NDF level in the diet. In contrast to the dry weight, the proportion of DM in the contents of both parts of the colon decreased linearly $(P<0.001$ or $P<0.01)$ although, in the case of the proximal colon, this change was curvilinearly $(P<0.001)$ related to the level of NDF intake. Both the linear and the quadratic components of the interaction between level of NDF intake and NDF source in the diet were highly significant $(P<0.001)$, indicating that the extent and the rate of decrease in the proportion of DM in the contents of the colon with level of NDF intake were strongly related to the source of NDF in the diet.

\section{Distribution of stained particles and polyethylene beads in the organs of the gastrointestinal tract}

Because the number of stained particles and polyethylene beads expressed as a proportion of the total varied widely within treatments, the results from each level of NDF intake for a particular fibre were pooled and the means and the ranges of the number of particles and beads within each segment of the gastrointestinal tract are presented in Table 3.

In general, the number of stained particles, from either the morning or the evening feed, found in the stomach and the small intestine represented only a small proportion of the total and, in the cases in which it exceeded the level of $0 \cdot 10$, it usually fluctuated over a wide range. Considerably higher proportions of stained particles from the morning feed were found in the contents of the caecum of the pigs given soya-bean hulls, pea hulls, maize hulls 


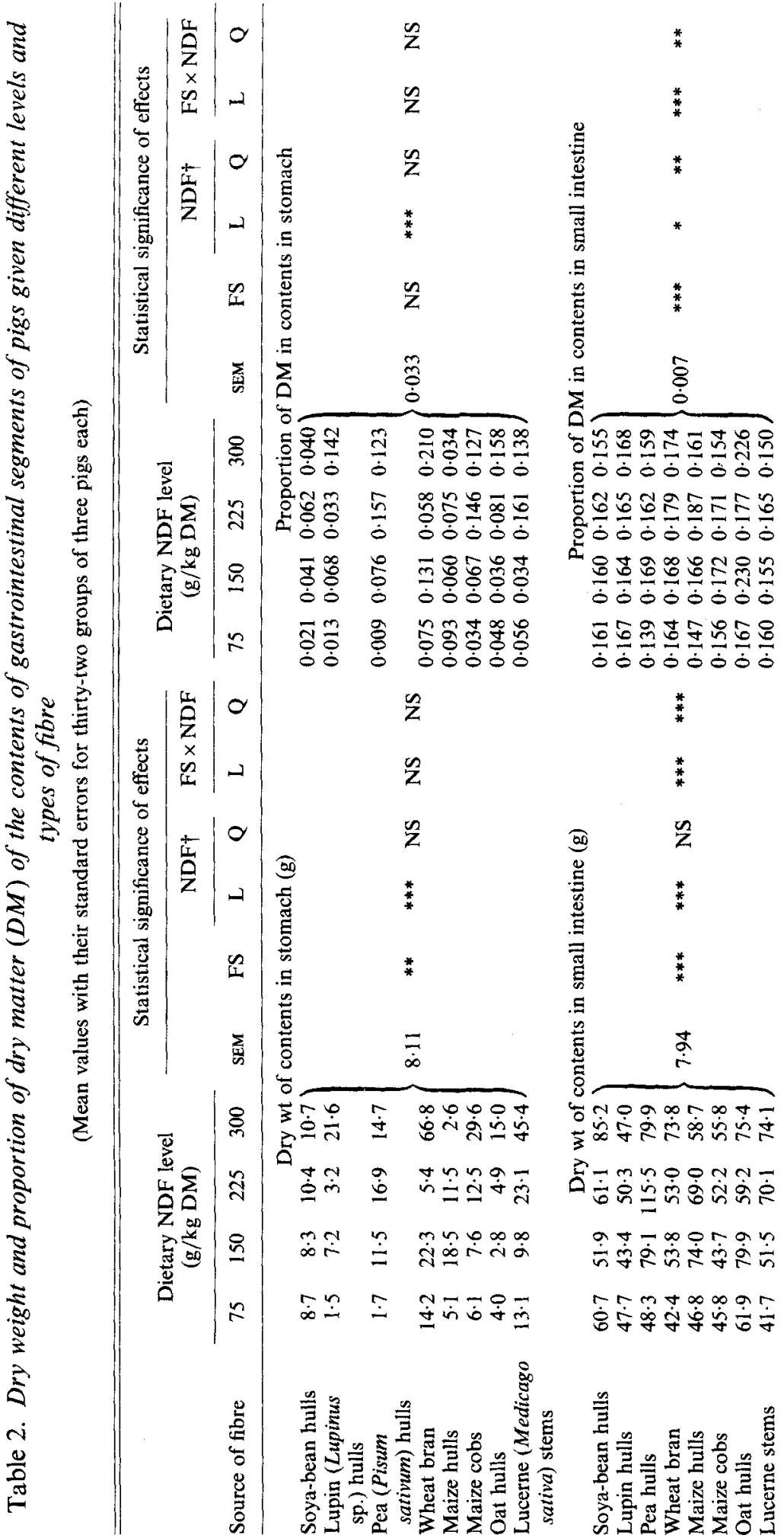



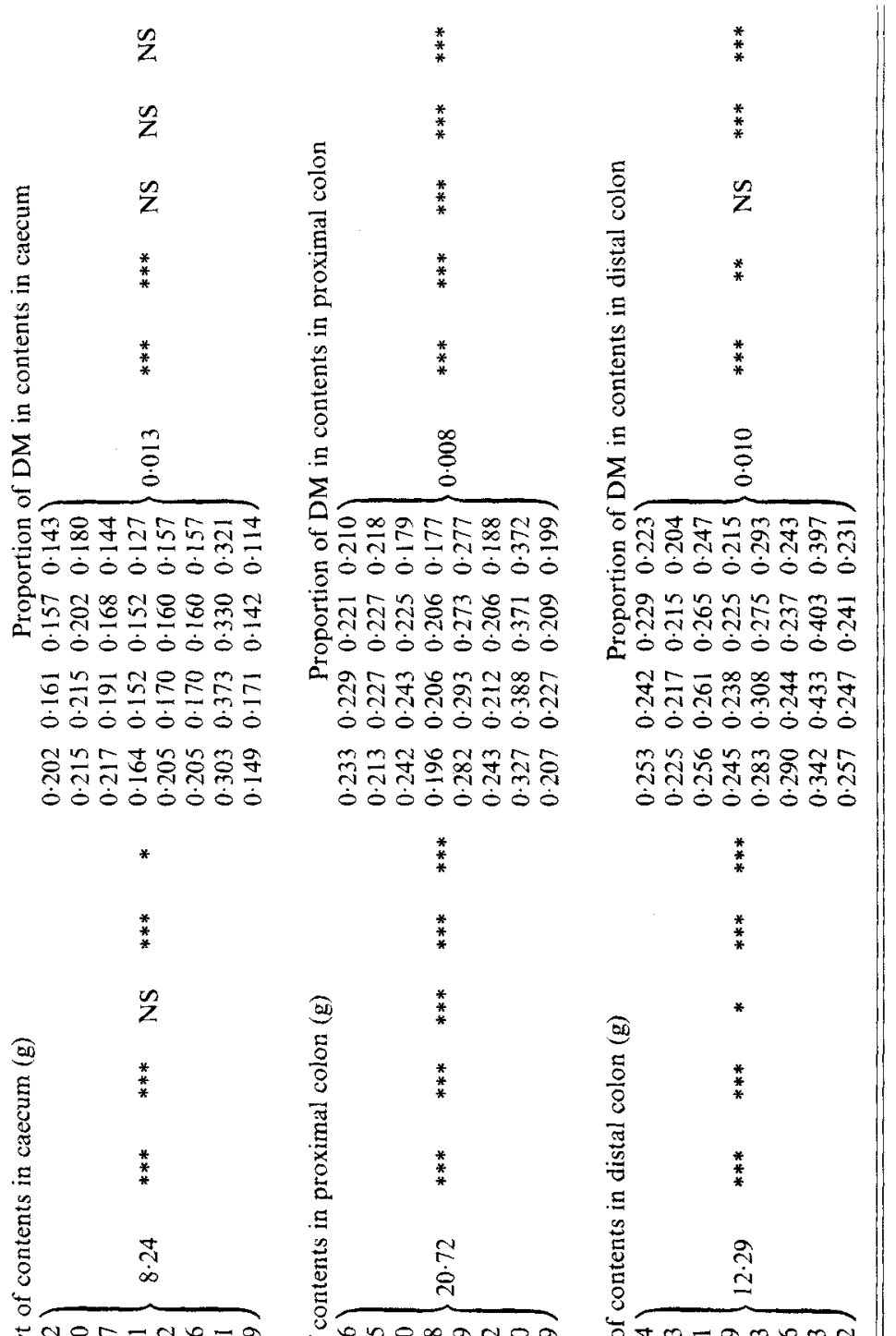

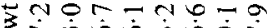

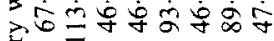
它

-

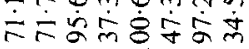
$-\infty m \infty$ o o

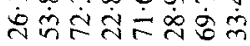
anon-na

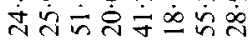

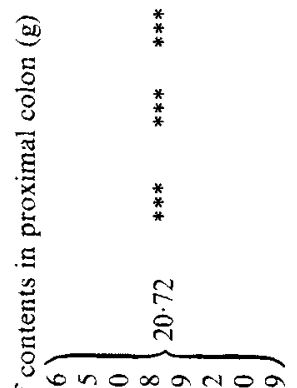

பั lagaron on

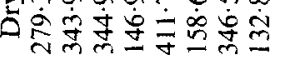

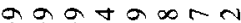

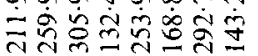

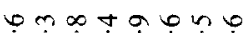
守守宁守穴它
00000000

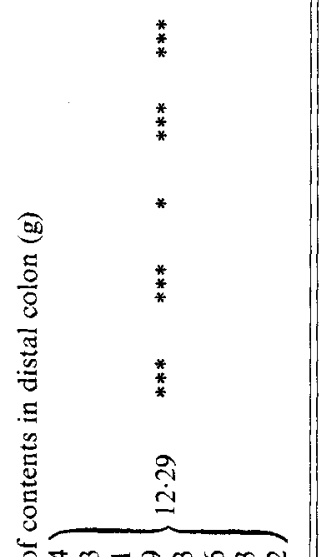

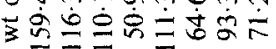
勾 $n-N-m+r$

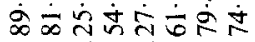
- о mamtot

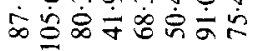
rutor.

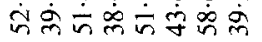

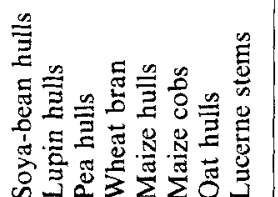




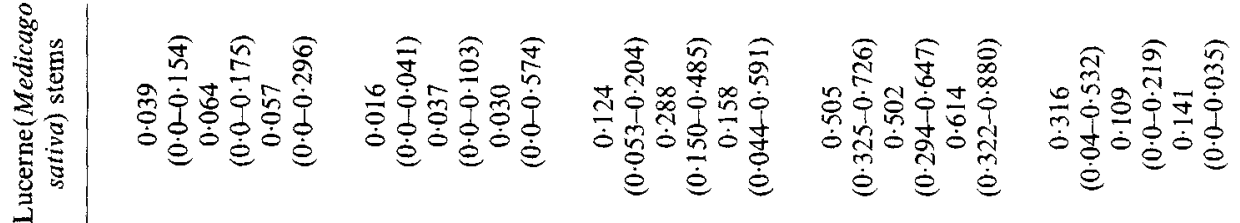

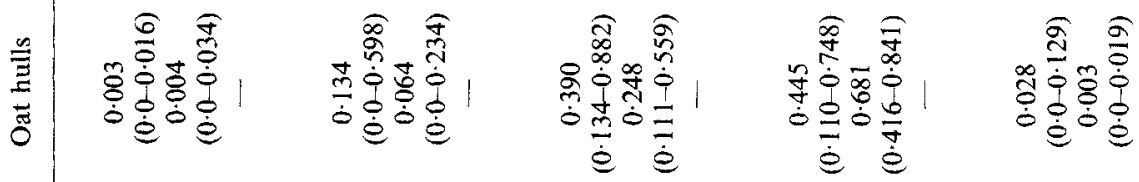

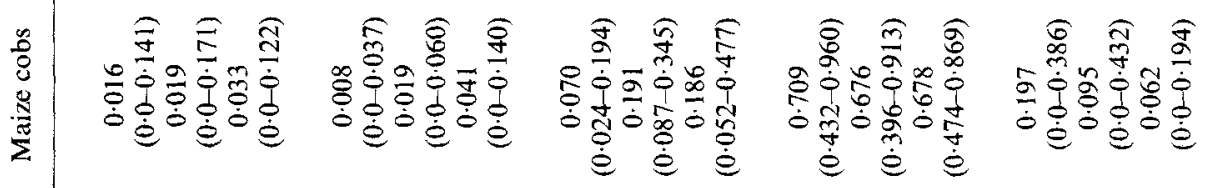

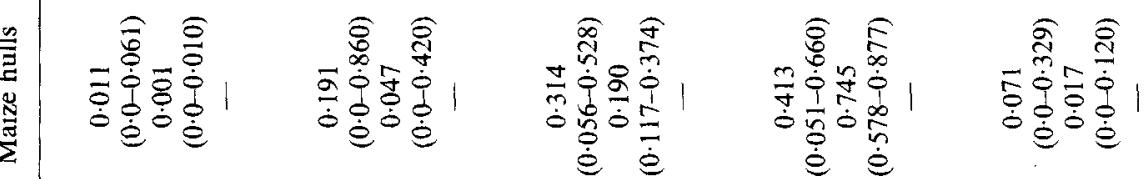

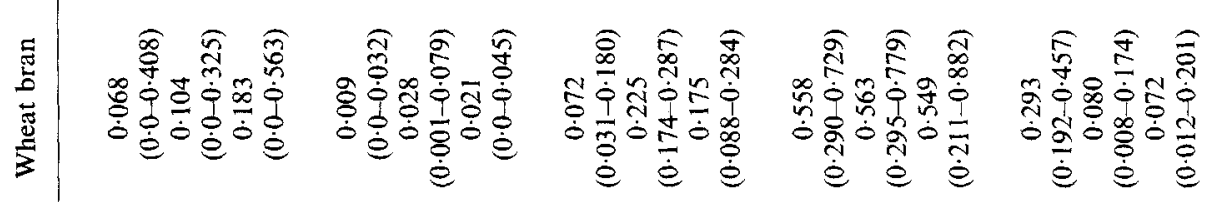

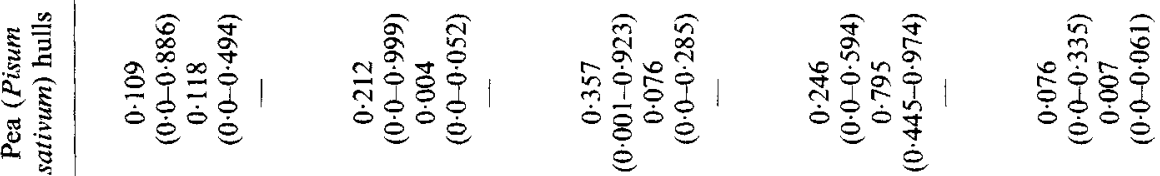

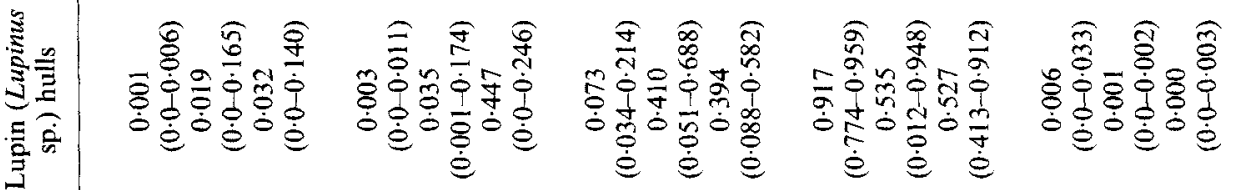

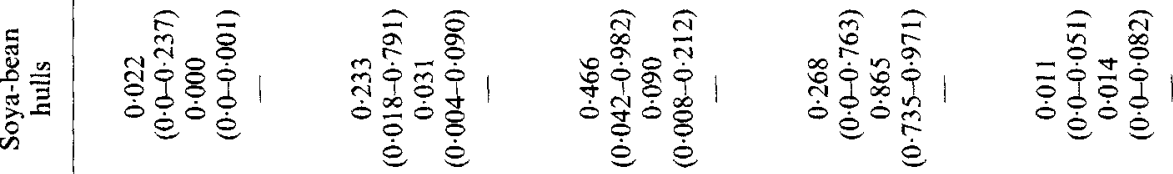

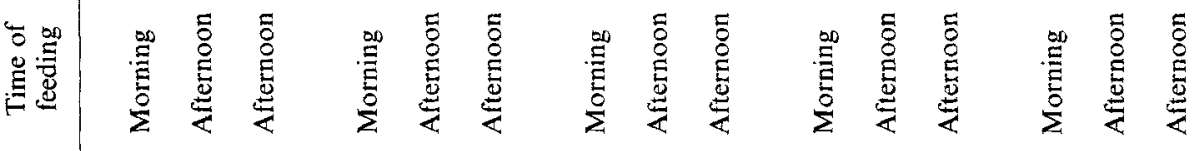

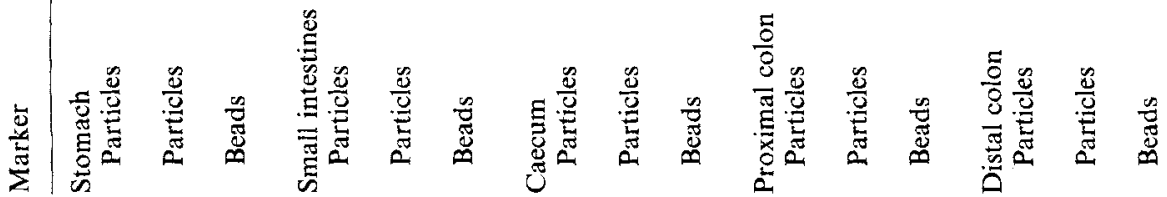


and oat hulls than in the caecum of the pigs given the rest of the fibres. Except for the pigs given soya-bean hulls, lupin hulls and pea hulls in which the number of particles from the evening feed found in the caecum represented $0.09,0.41$ and 0.08 of the total respectively, the mean numbers of particles in the caecum of the pigs given the rest of the fibres were similar, ranging from 0.19 to 0.29 of the total. In the pigs given soya-bean hulls, pea hulls, maize hulls and oat hulls, the number of stained particles from the morning feed in the proximal and the distal colons ranged from 0.28 to 0.48 of the total while, in the pigs given the rest of the fibres, it ranged from 0.78 to 0.92 . In the latter groups of pigs, $0.62-0.99$ of the total number of stained particles in the proximal and the distal colons were found in the proximal colon. With the meal given in the evening, the number of stained particles in the proximal colon of the pigs given soya-bean hulls, pea hulls and maize hulls ranged from 0.75 to 0.87 of the total while that of the pigs given the rest of the fibres, ranged from 0.50 to 0.68 of the total. Only a small number of stained particles from the evening feed, usually less than $0 \cdot 10$ of the total, were found in the distal part of the colon. The proportions of polyethylene beads usually approximated rather closely the proportions of the stained particles from the evening feed in individual segments of the gastrointestinal tract.

\section{DISCUSSION}

The tendency, in the present study, for an increased weight of the gastrointestinal organs, particularly that of the colon, with increased intakes by the pigs of NDF, is consistent with earlier reports in the literature (Coey \& Robinson, 1954; Wussow \& Weniger, 1954; Bohman et al. 1955; Kass et al. 1980, Pond et al. 1981) although, in the work reported by Hochstetler et al. (1959), Cunningham et al. (1961) and Gargallo \& Zimmerman (1980) very little or no change occurred in the organs of the gastrointestinal tract of pigs given diets containing increased levels of lucerne, wheat bran, cellulose or sunflower hulls. These differences between the different investigations might have been due, at least in part, to differences in the genotype of the pigs used (Petersson et al. 1979; Pond et al. 1981), to individual variation among pigs in the ability to utilize fibre (King \& Taverner, 1975) and differences in the source (Forbes \& Hamilton, 1952; Hochstetler et al. 1959; Komai \& Kimura, 1980; Van Soest, 1984) and in the amount of fibre ingested by the pigs.

There is now substantial evidence that microbial fermentation of dietary fibre in the large intestine of pigs results in the production of significant quantities of volatile fatty acids (VFA) (Argenzio et al. 1975; Argenzio \& Southworth, 1975). These VFA provide 0.05-0.28 of the pigs' energy requirement depending on the quantity of fermentable substrate available to the micro-organisms in the pigs' large intestine (Friend et al. 1964; Farrell \& Johnson, 1972; Imoto \& Namioka, 1978 a; Kass et al. 1980). Therefore, if pigs could become adapted to a type of digestion that increases their ability to utilize energy from dietary fibre, it would appear that parts of the digestive tract would be enlarged by increasing in length or weight or both. Brown et al. (1979) reported that the weight or the length, or both, of the small intestine, the caecum and the colon were significantly greater in rats fed on a high-fibre (pectin) diet than in either those given the conventional pelleted diet or fibre-free basal diet. Histological examination of small-intestine segments revealed that muscle layer thickness of the small intestine was greater in the pectin-fed rats. This led the investigators to the suggestion that, in part, the increased muscle layer thickness was the cause for the increased weight of the small intestine and probably the weights of the caecum and the colon. The nature of the increased organ weight in the present study has not been determined but it is possibly due to the same reason.

Feeding of high-fibre diets to simple-stomached animals may also be responsible for morphological changes in the intestines other than the muscle layer thickness (number and 
height of villi; crypt:villus ratio), functional changes (nutrient absorption capacity) and biochemical changes (mucosal protein, DNA, and enzyme activity) in the digestive tract but these are not easily obtainable measurements (Brown et al. 1979; Komai \& Kimura, 1980).

The length of the small intestine as well as that of the distal colon was unaffected by the level of NDF intake. In contrast, there was an increase in the length of the caecum with increasing NDF intake, this increase being more marked in the pigs given lupin hulls and lucerne stems. This difference in response to graded intakes of NDF of the caecum, the small intestine and the colon cannot be adequately explained with the present findings alone. Perhaps the rapid passage of the food from the stomach and small intestine leading to an accumulation of food in the caecum, exceeding its normal capacity, caused a longitudinal enlargement of the caecum of the pigs. Since the length of the small intestine and the total colon was only slightly affected by the level of NDF intake, the increase in the weights of these segments could have resulted from either a hypertrophy of the wall or an increase in their diameter.

In general, changes in the size and weight of gastrointestinal organs are believed to reflect a hypertrophy of particular tissues of the organ in response to the increased amount of work performed by these organs in drying, mixing, shaping, moving and expelling large amounts of undigested dietary residues (Friedman, 1953; Fischer, 1957; Dowling et al. 1967; Araujo, 1978; Brown et al. 1979). The fact that inclusion in the pig diet of lupin hulls, which even at the higher levels of NDF intake had a relatively small volume, resulted in changes in some of the gastrointestinal organs similar to those resulting from the feeding of the much bulkier lucerne stems, can be partly explained by the formation of a thick paste in the gastrointestinal organs of the pigs given lupin hulls. This presumably required more effort by the colon in order to dry and propel the residue from the lupin hulls toward the rectum.

The amount of DM in the stomach and the small intestine of the pigs from either the morning or the evening feed, as indicated by the numbers of stained particles in these organs, represented only a small proportion of the total amount of DM in the alimentary tract. In addition, only a small proportion of the DM in the small intestine was of fibre origin and this was usually found close to the ileo-caecal junction. Thus it appears that the fibrous part of the diet travelled very fast through the stomach and the small intestine indicating that the role of these organs in fibre digestion is very limited. Nevertheless, disappearance of dietary hemicellulose, presumably due to enzymic hydrolysis, but not of cellulose, in the organs of the gastrointestinal tract anterior to the caecum has been reported by Keys \& DeBarthe (1974) and Kass et al. (1980).

It has been reported (Lepkovsky et al. 1957) that the small intestine regulates rather closely the proportion of moisture in its contents when a homogenous diet is given. As indicated by the relatively constant proportions of DM in the contents of the small intestine of the pigs given different types and amounts of fibre, this also seems to be true when the diet contains a variety of fibres at different levels.

The large intestine, including the caecum, contained the major part of the undigested residues and the stained particles from both the morning and the evening meals. Increased weights of the contents in the large intestine of pigs, in response to increased levels of wheat bran or cellulose in the diet, were observed by Hochstetler et al. (1959) and Cunningham et al. (1961) although, in the latter report, the differences between the low- and high-fibre diet were not statistically significant. The fact that it took less than $18 \mathrm{~h}$ for the bulk of the undigested residues from the evening meal to reach the upper parts of the large intestine, while the time required for $95 \%$ of the undigested residues of a single meal to be excreted in the faeces ranged between 50 and $100 \mathrm{~h}$ for the different fibres (Stanogias \& Pearce, 1985), suggests that this section of the alimentary tract has a major influence on the rate of passage 
of digesta. Retention time of the digesta in the large intestine of pigs of about $30 \mathrm{~h}$ has been reported previously (Hecker \& Grovum, 1975). Usually, greater quantities of DM were found in the proximal colon than in either the caecum or the distal colon.

There was a gradual increase in the proportion of DM in the contents between the caecum and distal colon, which indicates that the colon is the primary site of net water absorption as is the case for ruminants (Hecker \& Grovum, 1975) and most non-ruminant animals (Fell, 1969). As indicated by the markedly higher proportions of DM in the contents of the caecum and the colon of the pigs given oat hulls and maize hulls, as compared with those from the pigs given the rest of the fibres, there must be a relation between the dehydrating capacity of the colon and the ability of a particular type of fibre or diet to take up and hold water against the absorptive forces of the intestines. Indeed, in vitro tests have shown that oat hulls and maize hulls absorb and retain considerably less water compared with the rest of the fibre sources (G. Stanogias, unpublished results). Although cellulose has been implicated as the cell-wall component that influences mostly the ability of a fibre to hold water (McConnell et al. 1974) and the proportion of DM in the contents of the large intestine (Cooper \& Tyler, 1959), the present results do not seem to confirm this suggestion. On the contrary, it has been suggested that of the cell-wall components, pentosan can best predict faecal weight increase (Cummings et al. 1978) in man but gum arabic, which is rich in pentoses, was found to have little effect on faecal weight in man (Ross et al. 1983). It seems, therefore, that a combination of fibre properties including chemical structure of fibre, particle size and other physical properties and fermentability of a fibre are of great importance in this respect.

The present study shows that the organs of the gastrointestinal tract of the growing pig, particularly the large intestine, respond to increased intakes by the pig of fibre, even for short periods of time, by increasing their mass or length, or both, and the magnitude of this effect seems to be dependent on the type of fibre given. Other factors, however, such as products of the microbial breakdown of fibre and dietary components other than fibre, may also play an important role in altering the morphological and perhaps the functional and biochemical characteristics of the gastrointestinal organs and need to be examined in relation to dietary fibre.

\section{REFERENCES}

Araujo, P. E. (1978). Journal of Food Science 43, 1040-1042.

Argenzio, R. W. \& Southworth, M. (1975). American Journal of Physiology 228, 454460.

Argenzio, R. A., Southworth, M. \& Stevens, C. W. (1975), American Joumal of Physiology 226, 1043-1050.

Baird, D. M., McCampbell, H. C. \& Allison, J. R. (1970). Journal of Animal Science 31, 518-525.

Baird, D. M., McCampbell, H. C. \& Allison. J. R. (1975). Journal of Animal Science 41, 1039-1047.

Bohman, V. R., Hunter, J. E. \& McCormick, J. (1955). Journal of Animal Science 14, 499-506.

Brown, R. C., Kelleher, J. \& Losowsky, H. S. (1979). British Journal of Nutrition 42, 357-365.

Coey, W. E. \& Robinson, K. L. (1954). Joumal of Agricultural Science, Cambridge 45, 41-47.

Cole, D. J. A., Duckworth, J. E. \& Holmes, W. (1967). Animal Production 9, 141-148.

Cooper, P. H. \& Tyler, C. (1959). Joumal of Agricultural Science, Cambridge 52, 322-339.

Cummings, J. H., Southgate, D. A. T., Branch, W., Houston, H., Jenkins, D. J. A. \& James, W. P. T. (1978). Lancet i, 5-9.

Cunningham, H. M., Friend, D. W. \& Nicholson, J. W. G. (1961). Canadian Journal of Animal Science 41, $120-125$.

Dowling, R. H., Riecken, E. O., Laws, J. W. \& Booth, C. C. (1967). Clinical Science 32, 1-9.

Farrell, D. J. \& Johnson, K. A. (1972). Animal Production 14, 209-217.

Fell, B. F. (1969). In Nutrition of Animals of Agricultural Importance, p. 295 [D. Cuthbertson, editor]. Oxford: Pergamon Press.

Fischer, J. E. (1957). American Journal of Physiology 188, 550-554.

Forbes, R. M. \& Hamilton, T. S. (1952). Journal of Animal Science 11, 480-490.

Friedman, M. H. F. (1953). Journal of the National Cancer Institute 13, 1035-1041. 
Friend, D. W., Nicholson, W. G. \& Cunningham, H. M. (1964). Canadian Journal of Animal Science 44, 303-308. Gargallo, J. \& Zimmerman, D. R. (1980). Journal of Animal Science 51 (Supplement 1), 198 (Abstract).

Hecker, J. F. \& Grovum, W. L. (1975). Australian Journal of Biological Sciences 28, 161-167.

Hochstetler, L. N., Hoefer, J. A., Pearson, A. M.\& Luecke, R. W. (1959). Journal of Animal Science 18, 1397-1404. Imoto, S. \& Namioka, S. (1978a). Journal of Animal Science 47, 467-478.

Kass, M. L., Van Soest, P. J., Pond, W. G., Lewis, B. \& McDowell, R. E. (1980). Journal of Animal Science 50, $175-191$.

Keys, J. E. Jr \& DeBarthe, J. V. (1974). Journal of Animal Science 39, 53-56.

King, R. H. \& Taverner, M. R. (1975). Animal Production 21, 275-284.

Komai, M. \& Kimura, S. (1980). Journal of Nutritional Science \& Vitaminology 26, 389-399.

Lepkovsky, S., Lyman, R., Fleming, D., Nagumo, M. \& Dimick, M. M. (1957). American Journal of Physiology 188, 327-331.

McConnell, A. A., Eastwood, M. A. \& Mitchell, W. D. (1974). Journal of the Science of Food \& Agriculture 25, $1457-1464$.

Petersson, H., Hakansson, J. \& Eriksson, S. (1979). Swedish Journal of Agricultural Research 9, 75-82.

Pond, W. G., Yen, J. T., Lindvall, R. N. \& Hill, D. (1981). Journal of Animal Science 51, 367-373.

Ross, H. McL., Eastwood, M. A., Brydon, W. G., Anderson, J. R. \& Anderson, D. H. W. (1983). American Journal of Clinical Nutrition 37, 368-375.

Snedecor, G. W. \& Cochran, W. G. (1973). Statistical Methods, 6th ed. Ames, Iowa: Iowa State University Press.

Stanogias, G. \& Pearce, G. R. (1985). British Journal of Nutrition 53, 513-530.

Van Soest, P. J. (1984). Proceedings of the Nutrition Society 43, 25-33.

Wierda, J. L. (1950). Anatomical Record 107, 221-223.

Wussow, W. \& Weniger, J. H. (1954). Archivs für Tierernährung 4, 151-181.

Younoszai, M. K., Adedoyin, M. \& Ranshaw, J. (1978). Journal of Nutrition 108, 341-350. 